Cita: Gómez-Carmona, C.D.; Bastida-Castillo, A.; García-Rubio, J.; Pino-Ortega, J.; Ibáñez, S.J. (2019). Influencia del resultado en las demandas de carga externa en baloncesto masculino de formación durante la competición oficial. Cuadernos de Psicología del Deporte, Vol 19(1), 262-274

\title{
Influencia del resultado en las demandas de carga externa en baloncesto masculino de formación durante la competición official
}

\section{Game outcome influence in external load demands in youth male basketball during official competition}

\section{Influência dos resultados sobre a demanda de carga externa em basquetebol júnior masculino durante a concorrência oficial}

\author{
Gómez Carmona, Carlos D. ${ }^{1}$, Bastida-Castillo, Alejandro ${ }^{2}$, García-Rubio, Javier ${ }^{1}$, Pino-Ortega, José2 \\ Ibáñez, Sergio J. ${ }^{1}$ \\ ${ }^{1}$ Grupo de Optimización del Entrenamiento y Rendimiento Deportivo (GOERD). Departamento de \\ Didáctica de la Expresión Musical, Plástica y Corporal. Facultad de Ciencias del Deporte. Universidad \\ de Extremadura, Cáceres (España); ${ }^{2}$ Departamento de Actividad Física y Deporte. Facultad de Ciencias \\ del Deporte. Universidad de Murcia, San Javier, Murcia (España).
}

Financiación: El autor Carlos D. Gómez Carmona es beneficiario de una beca del Ministerio de Educación, Cultura y Deporte (FPU17/00407). Este trabajo ha sido parcialmente subvencionado por la Ayuda a los Grupos de Investigación (GR18170) de la Junta de Extremadura (Consejería de Empleo e Infraestructuras); con la aportación de la Unión Europea a través de los Fondos Europeos de Desarrollo Regional (FEDER).

Conflicto de intereses: Trabajo desarrollado dentro del Grupo de Optimización del Entrenamiento y Rendimiento Deportivo (GOERD) de la Facultad de Ciencias del Deporte de la Universidad de Extremadura. Todos los autores hemos contribuido en la realización del manuscrito y certificamos que no ha sido publicado ni está en vías de consideración para su publicación en otra revista. Aceptamos las normas de publicación de la Revista. Asimismo, transferimos los derechos del presente trabajo a Cuadernos de Psicología del Deporte con ISSN 1989-5879, firmado por todos los autores. 


\section{Influencia del resultado en las demandas físicas en baloncesto formación}

\section{RESUMEN}

El estudio de las relaciones entre el marcador final y la eficacia técnico-táctica de jugadores y equipos está muy extendido. En cambio, no existen suficientes evidencias sobre si este fenómeno influye directamente en las demandas de carga externa durante la competición. El objetivo de la presente investigación fue analizar la influencia del marcador parcial en cada uno de los períodos de juego en las demandas de carga externa que presentan los jugadores de baloncesto masculino de formación. 96 jugadores de baloncesto sub-18 de nivel élite masculino pertenecientes a ocho equipos fueron registrados durante 13 partidos de la fase final del Adidas Next Generation Tournament 16-17 mediante dispositivos inerciales WIMU PRO ${ }^{\mathrm{TM}}$ a través de la tecnología de seguimiento de ultra-banda ancha (UWB). La prueba estadística T-Student de muestras independientes junto con el tamaño del efecto mediante d de Cohen fueron utilizados para el análisis comparativo en relación al marcador final del cuarto: (a) equilibrados $(<10$ puntos) y (b) desequilibrados ( $>10$ puntos). Se encuentran diferencias estadísticamente significativas en todas las variables de carga externa analizadas. Las mayores exigencias fueron realizadas en los partidos desequilibrados $(p<.01 ; t=1.93-3.32 ; d=0.42-0.64)$ encontrando las mayores diferencias en la variable impactos en todas las intensidades (3-5G, 5-8G, $>8 \mathrm{G}$ ). En conclusión, el marcador tiene una influencia directa en la carga externa que soportan los jugadores durante los partidos oficiales. Por tanto, es necesario reproducir estas situaciones durante los entrenamientos para someter a los jugadores a estímulos semejantes a los que se producen en la competición. Palabras clave: Baloncesto; rendimiento; resultado; dispositivos inerciales; ultra-banda ancha.

\section{ABSTRACT}

The analysis between the match status and the technical-tactical effectiveness of players and teams is very widespread. Instead, it has not enough evidence whether this phenomenon directly influences the external load demands during the competition. The objective of the present study is to analyse the influence of game outcome in the external load demands performed by youth male basketball players. 96 elite-level male U'18 basketball players of eight teams were registered during 13 matches of the Adidas Next Generation Tournament 16-17 final round using WIMU PRO ${ }^{\mathrm{TM}}$ inertial devices through ultra-wide band (UWB) tracking technology. The T-Student statistical test of independent samples with Cohen's d effect size were used for the comparison analysis in relation to the game outcome: (a) balanced ( $<10$ points) and (b) unbalanced ( $>10$ points). Statistically significant differences were found in the external load variables analysed. The higher load demands were performed in unbalanced matches $(p<.01$; $t=1.93-3.32 ; d=0.42-0.64)$, finding the greatest differences in impacts at all intensities (3-5G, 5-8G,> 8G). In conclusion, the score has a direct influence in the external load during official matches. Therefore, it is necessary to reproduce these situations during training to face the players with similar stimuli to the competition.

Keywords: Basketball; performance; game outcome; inertial devices; ultra-wide band.

\section{RESUMO}

O estudo da relação entre o escore final e a eficácia técnico-tática de jogadores e equipes é generalizado, mas ainda não há evidências suficientes sobre se esse fenômeno influencia diretamente as demandas externas de carga durante a competição. O objetivo da presente investigação foi analisar a influência do marcador parcial em cada um dos períodos de jogo nas demandas de carga externa apresentadas pelos jogadores júnior masculino de basquetebol. 96 jogadores sub-18 homens pertencentes a oito equipes de basquete de nível elite foram registrados durante 13 jogos da fase final do Adidas Next Generation Tournament 16-17 por dispositivos inerciais WIMU PRO ${ }^{\mathrm{TM}}$ (RealTrack Sistemas, Almeria, Espanha), obter a análise do tempo-movimento em estádios cobertos por tecnologia de ultrabanda larga (UWB). Os testes estatísticos T-Student para amostras independentes com o tamanho do efeito através de d de Cohen foram utilizados para a análise diferencial em relação a pontuação parcial: (a) equilibrado ( $<10$ pontos) e (b) não equilibrado (> 10 pontos). As diferenças estatisticamente significativas foram encontradas em todas as variáveis analisadas da carga externa, com as mais elevadas exigências sobre jogos com resultado não equilibrado $(p<.01 ; t=1.93-3.32 ; d=0.42-0.64)$, encontrando as principais diferenças nos impactos as todas suas gamas (3-5G, 5 $8 \mathrm{G},>8 \mathrm{G})$. Em conclusão, a pontuação é um fator determinante na carga externa que os jogadores suportam durante as partidas oficiais. Portanto, é necessário reproduzir essas situações durante o treinamento para submeter os jogadores a estímulos semelhantes aos que ocorrem na competição.

Palavras chave: Basquetebol; desempenho; resultado; dispositivos inerciais; ultra-banda larga. 


\section{Gómez-Carmona, C.D.; Bastida-Castillo, A.; García-Rubio, J.; Pino-Ortega, J.; Ibáñez, S.J.}

\section{INTRODUCCIÓN}

El baloncesto, que se enmarca dentro de los deportes colectivos de invasión, presenta una gran cantidad de acciones intermitentes de alta intensidad que resultan relevantes y determinan el éxito durante la competición (Stojanović et al., 2018). El registro y posterior análisis de la carga que soportan los jugadores durante la competición oficial aportan una información muy importante para comprender las demandas energéticas, cinemáticas y técnicas de los jugadores, con el objetivo de diseñar entrenamientos que optimicen el rendimiento en la competición (Montgomery, Pyne, y Minahan, 2010). Además, el aumento del número de partidos disputados y el elevado número de lesiones en este deporte (McLean, Strack, Russell, y Coutts, 2018), han ocasionado que en los últimos años el foco de atención se centre en tratar de describir de manera objetiva la carga de trabajo interna y externa de los jugadores (Drew y Finch, 2016) y su relación (Svilar, Castellano, y Jukic, 2018), para lo que el estudio de diferentes protocolos de entrenamiento y su influencia en el rendimiento deportivo es esencial (Freitas, Calleja-González, Carlos-Vivas, Marín-Cascales, y Alcaraz, 2018).

En los últimos años, la monitorización de la carga externa ha sido ampliamente abordada por los dispositivos inerciales en los deportes de exterior como el futbol, el rugby y el futbol australiano (Aughey, 2011; Cummins, Orr, O'Connor, y West, 2013). La cuantificación de las demandas externas en baloncesto mediante microtecnología presenta varias limitaciones, entre las que se incluye el análisis tiempo-movimiento en estadios cubiertos (Edwards et al., 2018). Sin embargo, esta problemática ha sido resuelta gracias a la tecnología de posicionamiento local de ultra banda-ancha (ultra-wide-band, UWB) la cual ha demostrado ser precisa y fiable para la monitorización de la posición de jugadores en condiciones de interior (Bastida-Castillo, Gómez Carmona, De la Cruz Sánchez, y Pino Ortega, 2018; Bastida-Castillo, Gómez-Carmona, Hernández, y Pino-Ortega, 2018; Leser, Schleindlhuber, Lyons, y Baca, 2014). Pero, la gran cantidad de variables que nos aportan, deben de ser previamente reducidas para una simplificación en su análisis (Svilar, Castellano, Jukic, y Casamichana, 2018). Por tanto, su registro se ha convertido en imprescindible para el análisis de la carga física, debido a su relación con los indicadores de rendimiento $y$ las variables situaciones que determinan el éxito en la competición (Gomes et al., 2017).

En este sentido, existe un amplio estudio sobre los indicadores de rendimiento en los equipos ganadores $\mathrm{y}$ perdedores en baloncesto, encontrando una influencia en los rebotes defensivos, el éxito en lanzamientos de dos y tres puntos, los tiros libres encestados, las pérdidas de balón, las asistencias y las faltas cometidas (Conte et al., 2018; García, Ibáñez, Gómez, y Sampaio, 2014; Gómez, Ibáñez, Parejo, y Furley, 2017). Además, el análisis de las variables situacionales es importante debido a que son factores determinantes en el rendimiento deportivo, entre las que se encuentran el acierto en acciones específicas en función del rol del jugador (Choi, Kim, Lee, Suh, y So, 2015; García-Rubio, Gómez, Cañadas, y Ibáñez, 2015), las características individuales de cada equipo (García, Ibáñez, Martínez De Santos, Leite, y Sampaio, 2013; Pérez-Sánchez, Salmerón-Gómez, y Ocaña-Peinado, 2018), y la evolución de la reglamentación (Ibáñez, García-Rubio, Gómez, y González-Espinosa, 2018). Aun así, todas estas variables están en relación a la especificidad de la población de estudio.

Por tanto, es necesario el estudio de la categoría U' 18 debido a: (a) relación específica entre las características antropométricas y el rendimiento en esprint, resistencia y capacidad de salto, obteniendo mayores valores que la categoría U'15 (Nikolaidis et al., 2015); (b) eficacia técnico-táctica, con mayor ratio de puntos por posesión, rebotes defensivos y eficacia en tiro de 2 y tiros libres como indicadores de rendimiento entre equipos ganadores y perdedores (Ibáñez, Sampaio, Sáenz-López, Giménez, y Janeira, 2003); (c) efecto de la edad relativa, el cual es más pronunciado en categoría U'16 y U'18 con respecto a categoría senior (Arrieta, Torres-Unda, Gil, y Irazusta, 2016; Ibáñez, Mazo, Nascimento, y García-Rubio, 2018); (d) desarrollo madurativo, encontrando un desarrollo individual en las capacidades neuromusculares, potencia aeróbica y flexibilidad con una evolución desde el último año de la etapa cadete (U'16) hasta el primer año de la etapa junior (U'18) (Calleja-González, Tobalina, Martínez-Santos, y Mejuto, 2015); y (e) efecto de la presión defensiva en la dinámica de juego (Leite et al., 2014), entre otras.

Aunque estos estudios previos ayudan a esclarecer los efectos de variables contextuales en los indicadores de 


\section{Influencia del resultado en las demandas físicas en baloncesto formación}

rendimiento en el baloncesto, y específicamente en baloncesto de formación, todos ellos están condicionados por la dinámica del marcador parcial en cada uno de los cuartos (Prieto, García, e Ibáñez, 2017). En este sentido, investigaciones recientes realizadas por García et al. (2014) y Lorenzo, Gómez, Ortega, Ibáñez, y Sampaio (2010) encuentran diferencias significativas en variables técnicas como rebotes, asistencias, eficacia en tiros de 2 puntos y rebotes defensivos entre partidos con marcador equilibrado (diferencia menor de 10 puntos) y partidos desequilibrados (diferencia superior a 10 puntos).

En definitiva, existe un amplio estudio de los indicadores de rendimiento, las variables situacionales y el marcador en la eficacia de las acciones técnicas. Hasta donde se conoce, no se encuentra ninguna investigación previa que haya analizado la influencia del marcador en relación a la carga externa que soportan los jugadores durante la competición oficial en baloncesto de formación. Por tanto, el presente estudio tiene como objetivo analizar la influencia del marcador parcial en cada uno de los cuartos en las demandas de carga externa que presentan los jugadores de baloncesto U'18 durante la fase final del Adidas Next Generation Tournament (ANGT) celebrado durante la temporada 2016-2017.

\section{MATERIAL Y MÉTODOS}

\section{Diseño}

El presente estudio empleó un diseño transversal con grupos naturales (Ato, López-García, y Benavente, 2013) para conocer las demandas de carga externa en función del resultado parcial en cada uno de los cuartos durante la fase final del ANGT 16-17, celebrado en Estambul.

\section{Participantes}

En esta investigación, 96 jugadores de baloncesto sub18 de nivel élite (edad: $17.6 \pm 0.8$ años; altura: $1.91 \pm 0.08 \mathrm{~m}$; masa corporal: $82.5 \pm 8.8 \mathrm{~kg}$ ) pertenecientes a 8 equipos masculinos participaron voluntariamente. Como requisito para el registro de cada uno de los jugadores, estos debían participar en el cuarto con una duración superior a seis minutos $(>60 \%$ del tiempo total del cuarto) (Sampaio, Drinkwater, y Leite, 2010), por tanto, en el análisis final se incluyeron 67 jugadores.
La altura de los sujetos fue medida con un tallímetro de pared durante una inspiración máxima (SECA, Hamburgo, Alemania). El peso corporal de los sujetos se obtuvo mediante un monitor de composición corporal modelo BC-601 (TANITA, Tokio, Japón). Tanto el cuerpo técnico como los jugadores fueron informados previamente de los detalles de la investigación y de sus posibles riesgos y beneficios, por lo que para ello se les suministró un consentimiento informado. En los jugadores menores de edad, el consentimiento fue suscrito por sus tutores legales. El estudio se desarrolló en base a las disposiciones éticas de la Declaración de Helsinki (2013), siendo aprobado por el Comité de Bioética de la Universidad (Número de registro 67/2017). La empresa Euroleague Properties S.A. autorizó todos los protocolos de actuación.

\section{Procedimiento y Material}

El estudio se llevó a cabo durante la celebración del ANGT 16-17 entre los días 18 y 21 de Mayo de 2017. En esta competición participaban los 8 mejores clubs U'18 de baloncesto de Europa clasificados durante la fase regular. Estos se dividían en dos grupos de cuatro equipos de forma aleatoria. Los combinados disputaban un partido contra cada equipo de su grupo. El mejor equipo de cada grupo se clasificaba para disputar la final del campeonato. El total de partidos analizados entre la fase de grupos y la final del campeonato fue de 13 partidos. La competición se disputó en el "Ahmet Comert Arena" y el "Sinan Erdem Dome" de la ciudad de Estambul, Turquía.

Para la monitorización de la carga externa durante la competición se utilizaron 24 dispositivos inerciales WIMU PRO ${ }^{\mathrm{TM}}$ (RealTrack Systems, Almería, España), los cuales fueron utilizados simultáneamente. Estos dispositivos disponen de tecnología de ultra-banda ancha (UWB) para la localización en situaciones de interior con una frecuencia de muestreo de $18 \mathrm{~Hz}$. La precisión y fiabilidad de la tecnología de seguimiento UWB para el análisis posicional (coordenadas, $\%$ TEM, $\mathrm{x}=1.00 \%$; $\mathrm{y}=1.00 \%$ ) (Bastida-Castillo, Gómez-Carmona, Hernández et al., 2018) y para el análisis tiempomovimiento $\quad(\% T E M=1.19 \%) \quad$ (Bastida-Castillo, Gómez-Carmona, De la Cruz Sánchez et al., 2018), además del registro de la carga neuromuscular a través de los acelerómetros del dispositivo (Bias $=-0.04-$ 


\section{Gómez-Carmona, C.D.; Bastida-Castillo, A.; García-Rubio, J.; Pino-Ortega, J.; Ibáñez, S.J.}

$0.02 \mathrm{~g} ; r=0.99$ - 1.00) (Gómez-Carmona, BastidaCastillo, García-Rubio, Ibáñez, y Pino-Ortega, 2018) han sido evaluados recientemente, obteniendo unos valores muy elevados.

Para crear el marco de referencia donde los dispositivos serían registrados, seis antenas fueron posicionadas fuera de los límites del campo a una distancia entre 4-5 metros respecto a las líneas de fondo y banda y a una altura entre 3 y 4 metros para conseguir una total cobertura de la pista. Para una correcta precisión y fiabilidad de la localización, las antenas y los dispositivos fueron conectados $\mathrm{y}$ calibrados siguiendo las instrucciones del fabricante. Para evaluar la precisión, se realizó un recorrido alrededor del perímetro de la pista que posteriormente fue proyectado en el software S PRO ${ }^{\mathrm{TM}}$ (RealTrack Systems, Almería, España). Este marco de referencia creado fue comparado respecto a las medidas de una pista oficial $(28 \times 15 \mathrm{~m})$.

Además, el dispositivo WIMU $\mathrm{PRO}^{\mathrm{TM}}$ dispone de diferentes sensores (acelerómetros, giroscopios, magnetómetro, chip GNSS y UWB, Ant+, Bluetooth, entre otros) para el registro de diferentes variables cinemáticas, fisiológicas y neuromusculares. También, cuentan con un microprocesador propio, memoria flash de $8 \mathrm{~GB}$ y una interfaz USB de alta velocidad con la finalidad de grabar, almacenar y descargar los datos. El dispositivo es alimentado por una batería interna con 4 horas de autonomía, tiene 70 gramos de peso y una dimensión de $81 \times 45$ x 16 milímetros. Para su colocación, 10 minutos antes del inicio del calentamiento previo al partido, se citaron a los jugadores y los dispositivos fueron ubicados mediante un arnés específico a la altura de la línea interescapular (Akenhead y Nassis, 2016), siendo fijados anatómicamente a cada jugador.

\section{Variables}

Se determinaron como variables independientes la diferencia de marcador en cada uno de los cuartos disputados, clasificándose en partido disputado (diferencia menor de 10 puntos) y partido desequilibrado (diferencia mayor de 10 puntos) (Lorenzo et al., 2010). Así mismo, las variables dependientes registradas cuantificaban la carga externa realizada por el jugador, analizando las siguientes que se describen en la tabla 1: distancia total
(Dellaserra, Gao, y Ransdell, 2014), distancia a alta intensidad (Stojanović et al., 2018), aceleraciones y deceleraciones (Svilar, Castellano, Jukic, et al., 2018), impactos a diferentes rangos $(3-5,5-8,>8 \mathrm{G})$ (Cunniffe, Proctor, Baker, y Davies, 2009), pasos y saltos (Reina, García-Rubio, Feu, y Ibáñez, 2018), PlayerLoad y potencia metabólica (Reche-Soto et al., 2018).

\begin{tabular}{|c|c|c|c|}
\hline Variable & Acrónimo & Unidad & Descripción \\
\hline Distancia total & DT & metros $(\mathrm{m})$ & Distancia total recorrida \\
\hline $\begin{array}{l}\text { Distancia Alta } \\
\text { Intensidad }\end{array}$ & HIA & metros (m) & $\begin{array}{l}\text { Distancia total recorrida a una } \\
\text { velocidad superior a } 16 \mathrm{~km} / \mathrm{h}\end{array}$ \\
\hline Aceleraciones & Acc & número (n) & $\begin{array}{l}\text { Total de cambios de velocidad } \\
\text { positivos }\end{array}$ \\
\hline Deceleraciones & Dec & número (n) & $\begin{array}{l}\text { Total de cambios de velocidad } \\
\text { negativos }\end{array}$ \\
\hline Impactos 3-5 G & {$[3-5 \mathrm{G}] \mathrm{Imp}$} & número $(\mathrm{n})$ & $\begin{array}{l}\text { Número total de impactos a baja } \\
\text { intensidad }\end{array}$ \\
\hline Impactos 5-8 G & {$[5-8 \mathrm{G}] \mathrm{Imp}$} & número (n) & $\begin{array}{l}\text { Número total de impactos a mediana } \\
\text { intensidad }\end{array}$ \\
\hline Impactos $>8 \mathrm{G}$ & {$[>8 \mathrm{G}] \mathrm{Imp}$} & número (n) & $\begin{array}{l}\text { Número total de impactos a alta } \\
\text { intensidad. }\end{array}$ \\
\hline Pasos & Pasos & número (n) & Número total de pasos. \\
\hline Saltos & Saltos & número $(\mathrm{n})$ & Número total de saltos. \\
\hline PlayerLoad & PL & $\begin{array}{l}\text { unidades } \\
\text { arbitrarias (a.u.) }\end{array}$ & $\begin{array}{l}\text { Carga acumulada resultante de la } \\
\text { aceleración en los } 3 \text { ejes. }\end{array}$ \\
\hline $\begin{array}{l}\text { Potencia } \\
\text { Metabólica }\end{array}$ & PM & $\begin{array}{l}\text { watios } / \text { kilogramo } \\
(\mathrm{W} / \mathrm{kg})\end{array}$ & $\begin{array}{l}\text { Gasto metabólico calculado como } \\
\text { producto de la velocidad instantánea } \\
(\mathrm{v}, \mathrm{m} \cdot \mathrm{s}-1) \text { y el coste energético en } \\
\text { relación al peso corporal y la } \\
\text { distancia recorrida }(\mathrm{Cr}, \mathrm{J} \cdot \mathrm{kg}-\mathrm{l} \cdot \mathrm{m}-\mathrm{m}-\mathrm{l})\end{array}$ \\
\hline
\end{tabular}

\section{Análisis estadístico}

En primer lugar, se realizó un análisis exploratorio mediante las pruebas de asunción de criterios. Se llevaron a cabo las pruebas de contraste del supuesto de normalidad (Shapiro-Wilk), y de contraste del supuesto de homocedasticidad (Prueba de Levene) obteniendo todas ellas una distribución normal (Field, 2013). Posteriormente, se realizó un análisis descriptivo donde los datos se muestran como media y desviación estándar (media $\pm \mathrm{DE}$ ) para describir todas las exigencias cinemáticas de los partidos analizados durante la competición en función del resultado final de cada uno de los cuartos. Para calcular la media de exigencias en cada uno de los cuartos únicamente se tuvieron en cuenta los jugadores que disputaron más de 6 minutos en cada cuarto analizado. Para comparar las diferencias en las variables analizadas en función del marcador parcial se utilizó la prueba $t$ de Student para muestras independientes. El nivel de significación se estableció con el valor de $p<0.05$.

Para calcular la magnitud de las diferencias entre entrenamientos y partidos se calculó el tamaño del efecto mediante la $d$ de Cohen, siendo acompañado por la probabilidad de efecto mediante las inferencias basadas en la magnitud (MBI). El estadístico $d$ fue 


\section{Influencia del resultado en las demandas físicas en baloncesto formación}

clasificado como efecto bajo (0-0.2), efecto pequeño (0.2-0.6), efecto moderado (0.6-1.2), efecto grande $(1.2-2.0)$ y efecto muy grande $(>2.0)$ (Hopkins, Marshall, Batterham, y Hanin, 2009). Para el análisis estadístico se utilizó el software Statistical Package of Social Science (versión 24, 2016; IBM Corp., IBM SPSS Statistics para MAC OS, Armonk, NY, EEUU) y para el diseño de los gráficos se utilizó el software GraphPad Prism (versión 7; GraphPad Software, La Jolla CA, EEUU).

\section{RESULTADOS}

En la figura 1 y 2 se muestra el análisis descriptivo e inferencial de las variables cinemáticas analizadas en la presente investigación en función del resultado parcial de los cuartos. En cada uno de los cuartos, los jugadores de baloncesto sub-18 de nivel élite cubren una distancia total de $864.39 \pm 181.58$ metros, de los cuales un $3.71 \pm 1.12 \%$ se producen a alta intensidad $(>16 \mathrm{~km} / \mathrm{h})$, realizan $724 \pm 144$ pasos, $8 \pm 3$ saltos, un poder metabólico de $4711.74 \pm 1947.68 \mathrm{~W} / \mathrm{kg}$ y reciben un PlayerLoad ${ }^{\mathrm{TM}}$ de $16.08 \pm 3.48$ a.u. Además, realizan un total de $198 \pm 53$ aceleraciones y $193 \pm 51$ deceleraciones, reciben un total de impactos de $107 \pm 26$ entre $3-5 \mathrm{G}, 18 \pm 6$ entre $5-8 \mathrm{G}$ y $3 \pm 1$ por encima de $8 \mathrm{G}$. Finalmente, en relación al análisis inferencial, se encuentran diferencias estadísticamente significativas en todas las variables de carga externa analizadas, siendo las exigencias cinemáticas mayores en los partidos desequilibrados respecto a los partidos equilibrados $(p>.01 ; t=1.93-3.32)$.
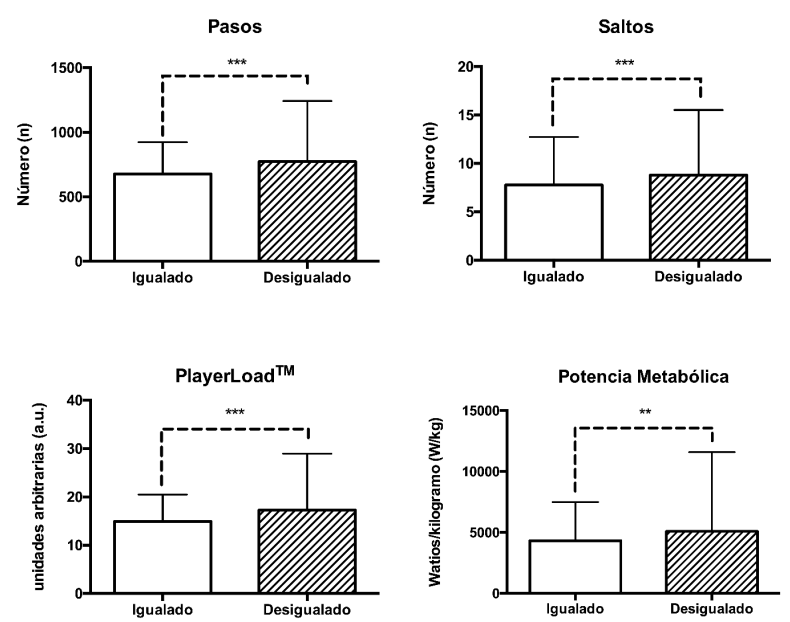

Figura 2. Representación gráfica y análisis diferencial de las variables pasos, saltos, PlayerLoad ${ }^{T M}$ y potencia metabólica registradas en la presente investigación en relación al resultado final en cada uno de los cuartos. ${ }^{*} p<.05 ; * * p<.01 ; * * * p<.001$.
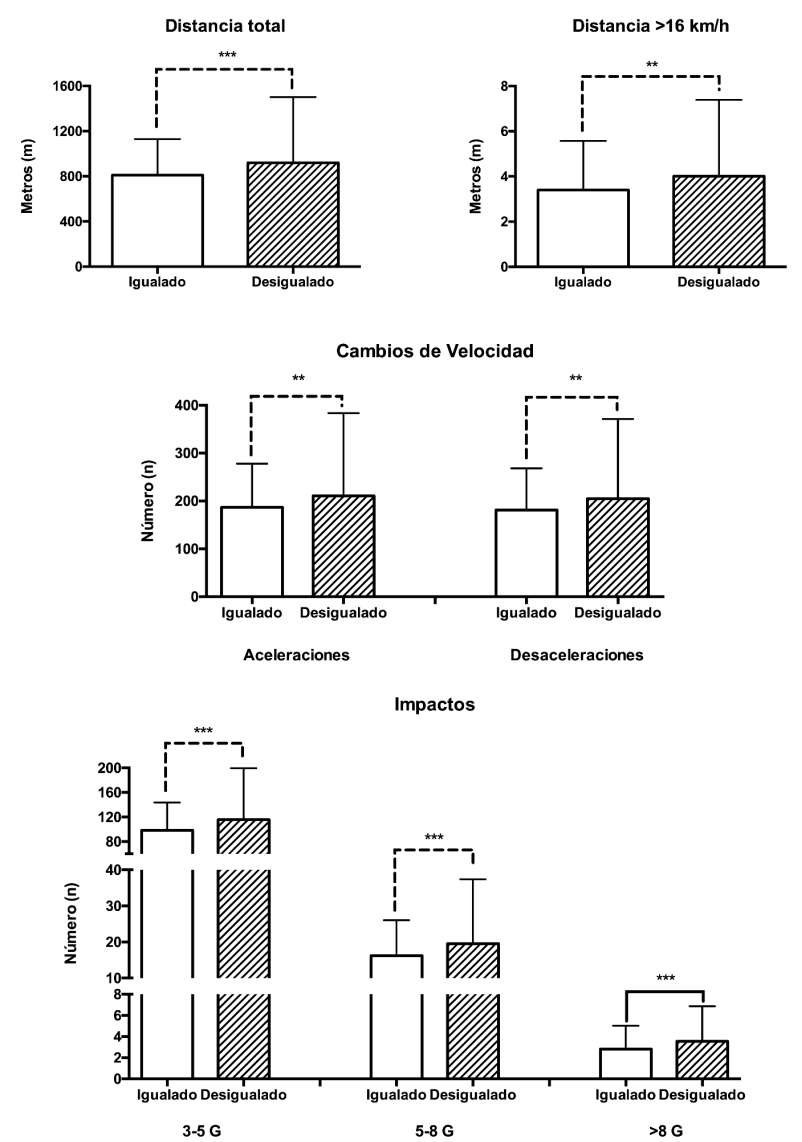

Figura 1. Representación gráfica y análisis diferencial de las variables distancia total, distancia a alta intensidad, cambios de velocidad (aceleraciones y desaceleraciones) e impactos registradas en la presente investigación en relación al resultado final en cada uno de los cuartos. ${ }^{*} p<.05 ; * *_{p}<.01 ; * * *_{p}<.001$.

En la figura 3, se muestra la magnitud de las diferencias en las variables analizadas en relación al resultado final de los cuartos mediante el estadístico $d$ de Cohen, encontrando un moderado tamaño del efecto $(d=.42-.64)$. Los mayores tamaños del efecto se encuentran en la variable Impactos en todos los rangos de intensidad $(3-5 \mathrm{G}, 5-8 \mathrm{G},>8 \mathrm{G})$, mientras que la menor magnitud de las diferencias se encontró en la variable Potencia Metabólica. 
Gómez-Carmona, C.D.; Bastida-Castillo, A.; García-Rubio, J.; Pino-Ortega, J.; Ibáñez, S.J.

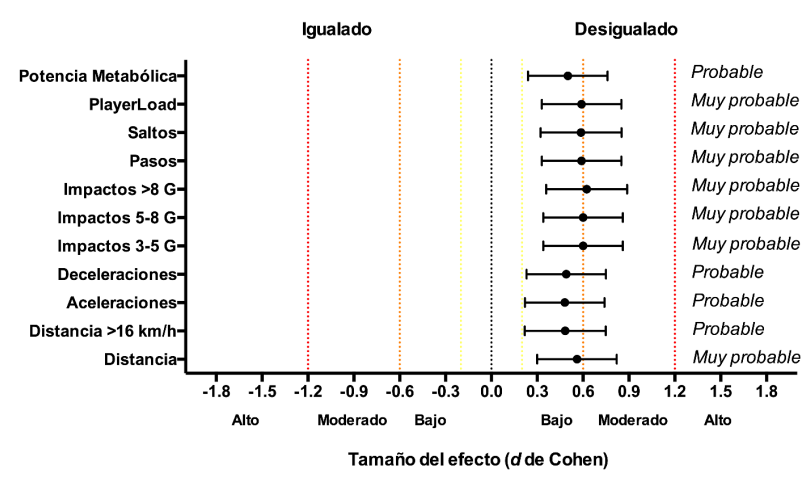

Figura 3. Representación gráfica de la magnitud de las diferencias mediante el estadístico d de Cohen junto con el IC 95\% de las variables analizadas en la presente investigación y la probabilidad de efecto mediante la inferencia basada en la magnitud (MBI) en función del resultado final de cada uno de los cuartos.

\section{DISCUSIÓN}

El presente estudio tuvo como objetivos, por un lado, describir las demandas de carga externa de jugadores de baloncesto de élite y, por otro, examinar las diferencias en estas exigencias en función del marcador final en cada uno de los períodos de juego. Los principales resultados obtenidos indican que el marcador es un factor que influye en las demandas de carga externa encontrando mayores exigencias cuando los partidos tienen un marcador desequilibrado, afectando en mayor medida a los impactos en todos los rangos de intensidad.

En cuanto al primer propósito, este estudio mostró que los participantes recorrieron $86.43 \pm 18.15 \mathrm{~m} / \mathrm{min}$, de los cuales un $3.71 \pm 1.12 \%$ se producen a alta intensidad, realizando cada minuto de juego de $72 \pm 14$ pasos, $0.8 \pm 0.2$ saltos, $19.8 \pm 5.3$ aceleraciones $\mathrm{y}$ $19.3 \pm 5.1$ deceleraciones. En la literatura científica, diferentes investigaciones han analizado este perfil en sexo masculino en categorías senior (Erčulj et al., 2008; Oba y Okuda, 2008; Puente, Abián-Vicén, Areces, López, y Del Coso, 2017; Scanlan, Dascombe, y Reaburn, 2011; Scanlan, Dascombe, Kidcaff, Peucker, y Dalbo, 2015) y junior (Abdelkrim, El Fazaa, El Ati, y Tabka, 2007; Hulka, Cuberek, y Belka, 2013; Oba y Okuda, 2008), además de en sexo femenino (Delextrat et al., 2015; Reina, García-Rubio, et al., 2018; Scanlan, Dascombe, Reaburn, y Dalbo, 2012). Se encuentran diferencias entre la carga obtenida en la literatura y entre estudios, encontrando perfiles específicos en función de las características individuales. Los resultados obtenidos en la presente investigación están en relación a los encontrados en categorías junior y senior élite, siendo menores los valores obtenidos en senior masculino de nivel inferior $\mathrm{y}$ en baloncesto femenino, tanto en distancia recorrida como en porcentaje de la misma a alta intensidad (Stojanović et al., 2018). Por tanto, el análisis de la competición permitirá identificar los patrones de movimiento específicos para cada nivel, edad y sexo, para a partir de los mismos, diseñar sesiones específicas de entrenamiento para conseguir una adaptación óptima a las exigencias del juego real.

Además de las demandas cinemáticas, los jugadores reportaron altos valores por minuto de juego en las variables poder metabólico de $471.17 \pm 194.76 \mathrm{~W} / \mathrm{kg}$, un PlayerLoad ${ }^{\mathrm{TM}}$ de $1.61 \pm 0.35$ a.u. y reciben $10.70 \pm 2.60$ impactos entre $3-5 \mathrm{G}, 1.78 \pm 0.56$ entre 5-8 G y $0.32 \pm 0.11$ por encima de $8 \mathrm{G}$ en cada uno de los cuartos. En la literatura científica, la variable más estudiada ha sido PlayerLoad ${ }^{\mathrm{TM}}$, la cual representa la suma vectorial de las aceleraciones realizadas por el cuerpo humano en los tres planos de movimiento (Montgomery et al., 2010; Peterson y Quiggle, 2017; Scanlan, Wen, Tucker, y Dalbo, 2014; Schelling y Torres, 2016). Solamente, Montgomery et al., (2010) analizó este aspecto en competición oficial en jugadores élite junior, encontrando un $\mathrm{PL}^{\mathrm{TM}}$ de $2.79 \pm 0.58$ a.u./min, siendo estos valores similares a los obtenidos en la presente investigación. Fuera de la competición, la variable PL ha sido analizada en situaciones controladas de 5vs5 en sesiones de entrenamiento, encontrando unos valores de $1.79 \pm 0.46$ a.u. $/ \mathrm{min}$ en 6 minutos de actividad en jugadores masculinos élite (Schelling y Torres, 2016), siendo los valores encontrados en 5 minutos de actividad a media pista mayores en jugadoras femeninas 3.48 \pm 0.86 a.u/min (Herrán, Usabiaga, y Castellano, 2017). Por lo tanto, se encuentra que las exigencias en cuanto a carga que soporta el cuerpo en los 3 ejes es similar cuando analizamos una población específica como jugadores sub-18 de baloncesto masculino de nivel élite. Para el trabajo y desarrollo de las sesiones de entrenamiento es muy importante tener en cuenta para un diseño específico de las tareas tanto la carga como los objetivos y contenidos que se desarrollan ya que provocan adaptaciones específicas que influirán en el rendimiento físico-técnico-táctico en baloncesto (Reina, González, Cañadas, y Ibáñez, 2018; Reina, Mancha, y Feu, 2017). 


\section{Influencia del resultado en las demandas físicas en baloncesto formación}

Con respecto al segundo objetivo analizado, el marcador ha influido significativamente en las exigencias de carga externa durante la competición, encontrando un moderado tamaño del efecto en partidos con marcador desequilibrado sobre los partidos con marcador equilibrado en todas las variables analizadas $(d=0.42-0.64)$. Desde el conocimiento de los autores, no se han encontrado estudios previos que hayan estudiado este fenómeno sobre la dinámica de las variables cinemáticas durante la competición, siendo únicamente estudiada esta dinámica para el análisis de la fatiga a lo largo de los cuartos (Abdelkrim et al., 2010; Oba y Okuda, 2008; Scanlan et al., 2011) sin tener en cuenta el marcador. Este aspecto si ha sido estudiado mediante análisis notacional sobre la eficacia en las acciones técnicotácticas, encontrando las peores estadísticas en los equipos perdedores en situaciones con marcador desequilibrado (Conte et al., 2018; García et al., 2014; Gómez et al., 2017; Lorenzo et al., 2010). Estas diferencias en los parámetros cinemáticos pueden deberse a una menor sincronización en los movimientos del equipo y por tanto una menor eficiencia en los desplazamientos (Folgado, Gonçalves, y Sampaio, 2018), a la influencia del ritmo de juego (Gómez et al., 2017; Romarís, Refoyo, y Lorenzo, 2014; Sampaio et al., 2010), o las diferentes dinámicas de comportamiento de las acciones técnicotácticas como una menor presión defensiva (Leite et al., 2014) o menor número de faltas y un mayor número de pérdidas de balón y de contraataques (García et al., 2014).

Por tanto, los diferentes escenarios que se producen durante la competición en relación al marcador (equilibrado vs desequilibrado) producen demandas heterogéneas a nivel físico que deben de ser analizadas (Gomes et al., 2017; Svilar, Castellano, Jukic, et al., 2018) y posteriormente reproducidas durante las sesiones de entrenamiento para conseguir una adaptación óptima del jugador ante estos estímulos (Freitas et al., 2018). Investigaciones recientes denominan a este fenómeno como "worst case scenarios", los cuales representan los periodos de corta duración de los partidos oficiales ( $<30$ segundos) donde se producen las máximas demandas, y que no pueden ser representados a través de la media total del entrenamiento/partido (Cunningham et al., 2018). Para su registro, nuevos sistemas de medición como los dispositivos inerciales son indispensables pues permiten un registro simultáneo de la carga externa, tanto en el análisis tiempo-movimiento mediante sistemas de localización indoor (LPM, UWB) (Bastida-Castillo, Gómez-Carmona, de la Cruz Sánchez et al., 2018; Ogris et al., 2012) y outdoor (Coutts y Duffield, 2010; Johnston, Watsford, Kelly, Pine, y Spurrs, 2014; Muñoz-López, Granero-Gil, Pino-Ortega, y De Hoyo, 2017), como en relación a la fuerza de la gravedad (acelerometría) (Dalen, Jørgen, Gertjan, Havard, y Ulrik, 2016; Schelling y Torres, 2016), y de la carga interna (Molina-Carmona, Gomez-Carmona, Bastida-Castillo, y Pino-Ortega, 2018) de una forma rápida y objetiva (Cummins et al., 2013). Actualmente, la gran heterogeneidad de sistemas de medición que se encuentran en el mercado para la medición de las exigencias (localización automática o semiautomática por video, dispositivos inerciales, dispositivos de seguimiento, etc.) provocan una limitación para la comparación entre estudios debido a los diferentes indicadores de carga o a los diferentes umbrales para la clasificación de las exigencias (Sweeting, Cormack, Morgan, y Aughey, 2017), siendo la tecnología UWB la más apropiada para el registro de la localización en espacios de interior por sus excelentes valores de precisión y fiabilidad $(<15 \mathrm{~cm})$ (Bastida-Castillo, GómezCarmona, Hernández et al., 2018; Leser et al., 2014).

Finalmente, esta investigación pone de manifiesto que es necesario un análisis de la competición desde un enfoque global debido a que no solamente los factores técnicos y tácticos son determinantes para conseguir un mayor éxito en la competición (García et al., 2014; Gómez, Lorenzo, Ibáñez, y Sampaio, 2013), sino también la adaptación física (Fox, Stanton, y Scanlan, 2018) y psicológica (Olmedilla y Dominguez-Igual, 2016) del jugador a los diferentes escenarios que se producen durante los partidos en relación al marcador. Para ello, es necesario emplear una combinación del registro de las variables de carga externa e interna mediante sistemas tecnológicos y su relación con respecto al análisis notacional (Scanlan, Fox, Borges, Tucker, y Dalbo, 2018; Svilar, Castellano, Jukic, et al., 2018) y el estado mental del deportista (Bohórquez Gómez-Millán y Checa Esquiva, 2017) para interpretar y relacionar todos los indicadores de rendimiento durante la competición. 


\section{Gómez-Carmona, C.D.; Bastida-Castillo, A.; García-Rubio, J.; Pino-Ortega, J.; Ibáñez, S.J.}

\section{CONCLUSIONES}

La diferencia de puntos que se produce al final de cada uno de los períodos de juego es un elemento determinante en la carga externa que soportan los jugadores durante los partidos oficiales. Por tanto, es necesario reproducir estas situaciones durante los entrenamientos para someter a los jugadores a estímulos físico-técnico-tácticos de igual o superior volumen e intensidad a los que se producen en la competición. Gracias a estas premisas, se podrá conseguir una adaptación óptima con el objetivo de mejorar el rendimiento deportivo del jugador de forma individual y del equipo a nivel colectivo. Futuras investigaciones deberán analizar el efecto de estas situaciones de entrenamiento con respecto a las exigencias competitivas y relacionar los indicadores de rendimiento individual con respecto a los indicadores de carga externa para conseguir una visión global de los indicadores de rendimiento de las modalidades deportivas, específicamente en baloncesto.

\section{REFERENCIAS}

1. Abdelkrim, N. B., Castagna, C., Jabri, I., Battikh, T., El Fazaa, S., y Ati, J. E. (2010). Activity Profile and Physiological Requirements of Junior Elite Basketball Players in Relation to AerobicAnaerobic Fitness: Journal of Strength and Conditioning Research, 24(9), 2330-2342. https://doi.org/10.1519/JSC.0b013e3181e381c1

2. Abdelkrim, N. B., El Fazaa, S., El Ati, J., y Tabka, Z. (2007). Time-motion analysis and physiological data of elite under-19-year-old basketball players during competition. British Journal of Sports Medicine, 41(2), 69-75. https://doi.org/10.1136/bjsm.2006.032318

3. Akenhead, R., y Nassis, G. P. (2016). Training Load and Player Monitoring in High-Level Football: Current Practice and Perceptions. International Journal of Sports Physiology and Performance, 11(5), 587-593. https://doi.org/10.1123/ijspp.2015-0331

4. Arrieta, H., Torres-Unda, J., Gil, S. M., y Irazusta, J. (2016). Relative age effect and performance in the U16, U18 and U20 European Basketball Championships. Journal of Sports Sciences,
34(16),

1530-1534.

https://doi.org/10.1080/02640414.2015.112220

5. Ato, M., López-García, J. J., y Benavente, A. (2013). Un sistema de clasificación de los diseños de investigación en psicología. Anales de Psicología, 29(3), 1038-1059. https://doi.org/10.6018/analesps.29.3.178511

6. Aughey, R. J. (2011). Applications of GPS technologies to field sports. International Journal of Sports Physiology and Performance, 6(3), 295310.

7. Bastida-Castillo, A., Gómez-Carmona, C. D., De la Cruz Sánchez, E., y Pino-Ortega, J. (2018). Accuracy, intra- and inter-unit reliability, and comparison between GPS and UWB-based position-tracking systems used for time-motion analyses in soccer. European Journal of Sport Science, $\quad 18(4), \quad 450-457$. https://doi.org/10.1080/17461391.2018.1427796

8. Bastida-Castillo, A., Gómez-Carmona, C. D., Hernandez, A., y Pino-Ortega, J. (2018). Validez $\mathrm{y}$ fiabilidad de un dispositivo inercial (WIMU PROTM) para el análisis del posicionamiento en balonmano. E-Balonmano. com: Revista de Ciencias del Deporte, 14(1), 9-16.

9. Bohórquez Gómez-Millán, M. R., y Checa Esquiva, I. (2017). Diseño y validez de contenido de una entrevista para la evaluación psicológica de porteros de fútbol. Revista de Psicología Aplicada al Deporte y el Ejercicio Físico, 2(1), e3, 1-12. https://doi.org/10.5093/rpadef2017a3

10. Calleja-González, J., Tobalina, J. C., MartínezSantos, R., y Mejuto, G. (2015). Evolución de las capacidades físicas en jugadores jóvenes de baloncesto de medio nivel Evolution of physical characteristics in mid level young basketball players. Cuadernos de Psicología del Deporte, 15(3), 199-204.

11. Choi, D.-H., Kim, S.-M., Lee, J.-W., Suh, S.-H., y So, W.-Y. (2015). Winning Factors: How Players' Positional Offensive and Defensive Skills Affect Probability of Victory in the Korea Basketball League. International Journal of Sports Science \& Coaching, $\quad$ 10(2-3), 453-459. https://doi.org/10.1260/1747-9541.10.2-3.453 


\section{Influencia del resultado en las demandas físicas en baloncesto formación}

12. Conte, D., Tessitore, A., Gjullin, A., Mackinnon, D., Lupo, C., y Favero, T. (2018). Investigating the game-related statistics and tactical profile in NCAA division I men's basketball games. Biology of Sport. 35(2), 137-143. https://doi.org/10.5114/biolsport.2018.71602

13. Coutts, A. J., y Duffield, R. (2010). Validity and reliability of GPS devices for measuring movement demands of team sports. Journal of Science and Medicine in Sport, 13(1), 133-135. https://doi.org/10.1016/j.jsams.2008.09.015

14. Cummins, C., Orr, R., O’Connor, H., y West, C. (2013). Global Positioning Systems (GPS) and Microtechnology Sensors in Team Sports: A Systematic Review. Sports Medicine, 43(10), 1025-1042. https://doi.org/10.1007/s40279-0130069-2

15. Cunniffe, B., Proctor, W., Baker, J. S., y Davies, B. (2009). An evaluation of the physiological demands of elite rugby union using global positioning system tracking software. The Journal of Strength \& Conditioning Research, 23(4), 1195-1203.

16. Cunningham, D. J., Shearer, D. A., Carter, N., Drawer, S., Pollard, B., Bennett, M., ... Kilduff, L. P. (2018). Assessing worst case scenarios in movement demands derived from global positioning systems during international rugby union matches: Rolling averages versus fixed length epochs. PLoS ONE, 13(4), e0195197. https://doi.org/10.1371/journal.pone.0195197

17. Dalen, T., Jørgen, I., Gertjan, E., Havard, H. G., y Ulrik, W. (2016). Player load, acceleration, and deceleration during forty-five competitive matches of elite soccer. The Journal of Strength \& Conditioning Research, 30(2), 351-359. https://doi.org/10.1519/JSC.0000000000001063

18. Delextrat, A., Badiella, A., Saavedra, V., Matthew, D., Schelling, X., y Torres-Ronda, L. (2015). Match activity demands of elite Spanish female basketball players by playing position. International Journal of Performance Analysis in Sport, 15(2), 687-703. https://doi.org/10.1080/24748668.2015.11868824

19. Dellaserra, C. L., Gao, Y., y Ransdell, L. (2014). Use of Integrated Technology in Team Sports: A Review of Opportunities, Challenges, and Future
Directions For Athletes. Journal of Strength and Conditioning Research, 2(28), 556-573. https://doi.org/10.1519/JSC.0b013e3182a952fb

20. Drew, M. K., y Finch, C. F. (2016). The Relationship Between Training Load and Injury, Illness and Soreness: A Systematic and Literature Review. Sports Medicine, 46(6), 861-883. https://doi.org/10.1007/s40279-015-0459-8

21. Edwards, T., Spiteri, T., Piggott, B., Bonhotal, J., Haff, G. G., y Joyce, C. (2018). Monitoring and Managing Fatigue in Basketball. Sports, 6(1), 19. https://doi.org/10.3390/sports6010019

22. Erčulj, F., Dežman, B., Vučković, G., Perš, J., Perše, M., y Kristan, M. (2008). An analysis of basketball players' movements in the slovenian basketball league play-offs using the sagit tracking system. Physical Education and Sport, 6(1), 7584.

23. Folgado, H., Gonçalves, B., y Sampaio, J. (2018). Positional synchronization affects physical and physiological responses to preseason in professional football (soccer). Research in Sports Medicine, 26(1), 51-63. https://doi.org/10.1080/15438627.2017.1393754

24. Fox, J. L., Stanton, R., y Scanlan, A. T. (2018). A Comparison of Training and Competition Demands in Semiprofessional Male Basketball Players. Research Quarterly for Exercise and Sport, $\quad 89(1)$, 103-111. https://doi.org/10.1080/02701367.2017.1410693

25. Freitas, T. T., Calleja-González, J., Carlos-Vivas, J., Marín-Cascales, E., y Alcaraz, P. E. (2018). Short-term optimal load training vs a modified complex training in semi-professional basketball players. Journal of Sports Sciences, 37(4), 434442.

https://doi.org/10.1080/02640414.2018.1504618

26. García, J., Ibáñez, J. S., Gómez, A. M., y Sampaio, J. (2014). Basketball Game-related statistics discriminating ACB league teams according to game location, game outcome and final score differences. International Journal of Performance Analysis in Sport, 14(2), 443-452. https://doi.org/10.1080/24748668.2014.11868733

27. García, J., Ibáñez, S. J., Martinez De Santos, R., Leite, N., y Sampaio, J. (2013). Identifying 


\section{Gómez-Carmona, C.D.; Bastida-Castillo, A.; García-Rubio, J.; Pino-Ortega, J.; Ibáñez, S.J.}

Basketball Performance Indicators in Regular Season and Playoff Games. Journal of Human Kinetics, $36(1)$, 161-168. https://doi.org/10.2478/hukin-2013-0016

28. García-Rubio, J., Gómez, M. Á., Cañadas, M., y Ibáñez, J. S. (2015). Offensive Rating-Time coordination dynamics in basketball. Complex systems theory applied to Basketball. International Journal of Performance Analysis in Sport, 15(2), 513-526. https://doi.org/10.1080/24748668.2015.11868810

29. Gomes, J. H., Rebello-Mendes, R., Almeida, M. B. de, Zanetti, M. C., Leite, G. dos S., y FigueiraJúnior, A. J. (2017). Relationship between physical fitness and game-related statistics in elite professional basketball players: Regular season vs. playoffs. Motriz: Revista de Educação Física, 23(2), e101626. https://doi.org/10.1590/s1980$\underline{6574201700020004}$

30. Gómez, M. A., Ibáñez, S. J., Parejo, I., y Furley, P. (2017). The use of classification and regression tree when classifying winning and losing basketball teams. Kinesiology, 49(1), 47-56. https://doi.org/10.26582/k.49.1.9

31. Gómez, M. A., Lorenzo, A., Ibañez, S.-J., y Sampaio, J. (2013). Ball possession effectiveness in men's and women's elite basketball according to situational variables in different game periods. Journal of Sports Sciences, 31(14), 1578-1587. https://doi.org/10.1080/02640414.2013.792942

32. Gómez-Carmona, C. D., Bastida-Castillo, A., García-Rubio, J., Ibáñez, S. J., y Pino-Ortega, J. (2018). Static and dynamic reliability of WIMU $\mathrm{PRO}^{\mathrm{TM}}$ accelerometers according to anatomical placement. Proceedings of the Institution of Mechanical Engineers, Part P: Journal of Sports Engineering and Technology, Epub: Ahead of print. https://doi.org/10.1177/1754337118816922

33. Herrán, A., Usabiaga, O., y Castellano, J. (2017). Comparación del perfil físico entre $3 \times 3$ y $5 \times 5$ de baloncesto formativo / Physical Profile Comparison Between $3 \times 3$ and $5 \times 5$ Basketball Training. Revista Internacional de Medicina $y$ Ciencias de la Actividad Física y del Deporte, 67, 435-457.

https://doi.org/10.15366/rimcafd2017.67.003
34. Hopkins, W. G., Marshall, S. W., Batterham, A. M., y Hanin, J. (2009). Progressive Statistics for Studies in Sports Medicine and Exercise Science: Medicine \& Science in Sports \& Exercise, 41(1), 3-13. https://doi.org/10.1249/MSS.0b013e31818cb278

35. Hulka, K., Cuberek, R., y Belka, J. (2013). Heart rate and time-motion analyses in top junior players during basketball matches. Acta Gymnica, 43(3), 27-35. https://doi.org/10.5507/ag.2013.015

36. Ibañez, S. J., García-Rubio, J., Gómez, M. A., y Gonzalez-Espinosa, S. (2018). The impact of rule modifications on elite basketball teams' performance. Journal of Human Kinetics, 64, 181193. https://doi.org/10.1515/hukin-2017-0193

37. Ibáñez, S. J., Mazo, A., Nascimento, J., y GarcíaRubio, J. (2018). The Relative Age Effect in under-18 basketball: Effects on performance according to playing position. PLoS ONE, 13(7), e0200408.

https://doi.org/10.1371/journal.pone. 0200408

38. Ibáñez, S. J., Sampaio, J., Sáenz-López, P., Giménez, J., y Janeira, M. A. (2003). Game statistics discriminating the final outcome of junior world basketball championship matches (Portugal 1999). Journal of Human Movement Studies, 45, 1-19.

39. Johnston, R. J., Watsford, M. L., Kelly, S. J., Pine, M. J., y Spurrs, R. W. (2014). Validity and interunit reliability of $10 \mathrm{~Hz}$ and $15 \mathrm{~Hz}$ GPS units for assessing athlete movement demands. The Journal of Strength \& Conditioning Research, 28(6),

1649-1655. https://doi.org/10.1519/JSC.0000000000000323

40. Leite, N., Leser, R., Gonçalves, B., CallejaGonzalez, J., Baca, A., y Sampaio, J. (2014). Effect of Defensive Pressure on Movement Behaviour During an Under-18 Basketball Game. International Journal of Sports Medicine, 35(09), 743-748. https://doi.org/10.1055/s-0033-1363237

41. Leser, R., Schleindlhuber, A., Lyons, K., y Baca, A. (2014). Accuracy of an UWB-based position tracking system used for time-motion analyses in game sports. European Journal of Sport Science, 14(7), 635-642. https://doi.org/10.1080/17461391.2014.884167 


\section{Influencia del resultado en las demandas físicas en baloncesto formación}

42. Lorenzo, A., Gómez, M. Á., Ortega, E., Ibáñez, S. J., y Sampaio, J. (2010). Game related statistics which discriminate between winning and losing under-16 male basketball games. Journal of Sports Science \& Medicine, 9, 664-668.

43. McLean, B. D., Strack, D., Russell, J., y Coutts, A. J. (2018). Quantifying Physical Demands in the National Basketball Association (NBA): Challenges in Developing Best-Practice Models for Athlete Care and Performance. International Journal of Sports Physiology and Performance, 24, 1-22. https://doi.org/10.1123/ijspp.2018-0384

44. Molina-Carmona, I., Gómez-Carmona, C. D., Bastida-Castillo, A., y Pino-Ortega, J. (2018). Validez del dispositivo inercial WIMU PRO ${ }^{\mathrm{TM}}$ para el registro de la frecuencia cardíaca en un test de campo. Sport-TK: Revista Euroamericana de Ciencias del Deporte, 7, 81-86.

45. Montgomery, P. G., Pyne, D. B., y Minahan, C. L. (2010). The Physical and Physiological Demands of Basketball Training and Competition. International Journal of Sports Physiology and Performance, $\quad 5(1), \quad 75-86$. https://doi.org/10.1123/ijspp.5.1.75

46. Muñoz-López, A., Granero-Gil, P., Pino-Ortega, J., y De Hoyo, M. (2017). The validity and reliability of a 5-hz GPS device for quantifying athletes' sprints and movement demands specific to team sports. Journal of Human Sport and Exercise, $\quad 12(1), \quad 156-166$. https://doi.org/10.14198/jhse.2017.121.13

47. Nikolaidis, P. T., Asadi, A., Santos, E. J. A. M., Calleja-Gonzalez, J., Padulo, J., Chtourou, H., y Zemkova, E. (2015). Relationship of body mass status with running and jumping performances in young basketball players. Muscles, Ligaments and Tendons Journal, 5(3), 187-194. https://doi.org/10.11138/mltj/2015.5.3.187

48. Oba, W., y Okuda, T. (2008). A Cross-sectional Comparative Study of Movement Distances and Speed of the Players and a Ball in Basketball Game. International Journal of Sport and Health Science, $6, \quad$ 203-212. https://doi.org/10.5432/ijshs.IJSHS20080336

49. Ogris, G., Leser, R., Horsak, B., Kornfeind, P., Heller, M., y Baca, A. (2012). Accuracy of the LPM tracking system considering dynamic position changes. Journal of Sports Sciences, 30(14), 1503-1511. https://doi.org/10.1080/02640414.2012.712712

50. Olmedilla, A., y Dominguez-Igual, J. J. (2016). Entrenamiento psicológico para la mejora de la atención y la autoconfianza en un futbolista. Revista de Psicología Aplicada al Deporte y el Ejercicio Físico, 1(1), 1-10. https://doi.org/10.5093/rpadef2016a4

51. Pérez-Sánchez, J. M., Salmerón-Gómez, R., y Ocaña-Peinado, F. M. (2018). A Bayesian asymmetric logistic model of factors underlying team success in top-level basketball in Spain: Bayesian asymmetric logistic model. Statistica Neerlandica, Epub: Ahead of Print. https://doi.org/10.1111/stan.12127

52. Peterson, K. D., y Quiggle, G. T. (2017). Tensiomyographical responses to accelerometer loads in female collegiate basketball players. Journal of Sports Sciences, 35(23), 2334-2341. https://doi.org/10.1080/02640414.2016.1266378

53. Prieto, J., García, J., y Ibañez, S. J. (2017). Scoring coordination patterns in basketball international championships of national teams. Revista de Psicología Del Deporte, 26(Suppl 1), 27-32.

54. Puente, C., Abián-Vicén, J., Areces, F., López, R., y Del Coso, J. (2017). Physical and Physiological Demands of Experienced Male Basketball Players During a Competitive Game: Journal of Strength and Conditioning Research, 31(4), 956-962. https://doi.org/10.1519/JSC.0000000000001577

55. Reche-Soto, P., Cardona-Nieto, D., Diaz-Suarez, A., Bastida-Castillo, A., Gomez-Carmona, C., Garcia-Rubio, J., y Pino-Ortega, J. (2018). Player Load and Metabolic Power Dynamics as Load Quantifiers in Soccer. Journal of Human Kinetics, Epub: Ahead of Print. http://www.doi.org/10.2478/hukin-2018-0072

56. Reina, M., García-Rubio, J., Feu, S., y Ibáñez, S. J. (2018). Training and competition load monitoring and analysis of women's amateur basketball by playing position: approach study. Frontiers in Psychology, 9, 2689. https://doi.org/10.3389/fpsyg.2018.02689

57. Reina, M., González, S., Cañadas, M., y Ibáñez, S. J. (2018). Análise das variáveis pedagogicas na 


\section{Gómez-Carmona, C.D.; Bastida-Castillo, A.; García-Rubio, J.; Pino-Ortega, J.; Ibáñez, S.J.}

tarefas de small sided games e full game no basquetebol. Corpoconsciencia, 22(02), 1-13.

58. Reina, M., Mancha, D., y Feu, S. (2017). ¿Se entrena como se compite? Análisis de la carga en baloncesto femenino. Revista de Psicología del Deporte, 26(Suppl1), 9-13.

59. Romarís, I. U., Refoyo, I., y Lorenzo, J. (2014). Comparación de los ritmos de juego en Liga Femenina y ACB. Cuadernos de Psicología del Deporte, 16(2), 161-168.

60. Sampaio, J., Drinkwater, E. J., y Leite, N. M. (2010). Effects of season period, team quality, and playing time on basketball players' game-related statistics. European Journal of Sport Science, 10(2), 141-149. https://doi.org/10.1080/17461390903311935

61. Scanlan, A., Dascombe, B., y Reaburn, P. (2011). A comparison of the activity demands of elite and sub-elite Australian men's basketball competition. Journal of Sports Sciences, 29(11), 1153-1160. https://doi.org/10.1080/02640414.2011.582509

62. Scanlan, A. T., Dascombe, B. J., Kidcaff, A. P., Peucker, J. L., y Dalbo, V. J. (2015). GenderSpecific Activity Demands Experienced during Semiprofessional Basketball Game Play. International Journal of Sports Physiology and Performance, $\quad 10(5), \quad 618-625$. https://doi.org/10.1123/ijspp.2014-0407

63. Scanlan, A. T., Dascombe, B. J., Reaburn, P., y Dalbo, V. J. (2012). The physiological and activity demands experienced by Australian female basketball players during competition. Journal of Science and Medicine in Sport, 15(4), 341-347. https://doi.org/10.1016/j.jsams.2011.12.008

64. Scanlan, A. T., Fox, J. L., Borges, N. R., Tucker, P. S., y Dalbo, V. J. (2018). Temporal changes in physiological and performance responses across game-specific simulated basketball activity. Journal of Sport and Health Science, 7(2), 176182. https://doi.org/10.1016/j.jshs.2016.05.002

65. Scanlan, A. T., Wen, N., Tucker, P. S., y Dalbo, V. J. (2014). The relationships between internal and external training load models during basketball training. The Journal of Strength \& Conditioning Research, 28(9), 2397-2405. https://doi.org/10.1519/JSC.0000000000000458
66. Schelling, X., y Torres, L. (2016). Accelerometer Load Profiles for Basketball-Specific Drills in Elite Players. Journal of Sports Science and Medicine, 15, 585-591.

67. Stojanović, E., Stojiljković, N., Scanlan, A. T., Dalbo, V. J., Berkelmans, D. M., y Milanović, Z. (2018). The Activity Demands and Physiological Responses Encountered During Basketball Match-Play: A Systematic Review. Sports Medicine, 48(1), 111-135. https://doi.org/10.1007/s40279-017-0794-z

68. Svilar, L., Castellano, J., y Jukic, I. (2018). Load monitoring system in top-level basketball team: relationship between external and internal training load. Kinesiology, 50(1), 25-33. https://doi.org/10.26582/k.50.1.4

69. Svilar, L., Castellano, J., Jukic, I., y Casamichana, D. (2018). Positional Differences in Elite Basketball: Selecting Appropriate Training-Load Measures. International Journal of Sports Physiology and Performance, 13(7), 947-952. https://doi.org/10.1123/ijspp.2017-0534

70. Sweeting, A. J., Cormack, S. J., Morgan, S., y Aughey, R. J. (2017). When Is a Sprint a Sprint? A Review of the Analysis of Team-Sport Athlete Activity Profile. Frontiers in Physiology, 8, 432. https://doi.org/10.3389/fphys.2017.00432 